Instituto Internacional de Investigación y Desarrollo Tecnológico Educativo INDTEC, C.A. DOI: $h$ ttps://doi.org/10.29394/Scientific.issn.2542-2987.2020.5.15.18.350-361

OAI-PMH: http://www.indteca.com/ojs/index.php/Revista Scientific/oai

Ensayo Original / Original Essay

\title{
Aprendizaje de la Tecnología en la Etapa Escolar Venezolana
}

\author{
Autora: Luisana Sleny López Alvarado \\ Colegio Universitario de Administración y Mercadeo, CUAM \\ sleny 82@hotmail.com \\ San Felipe, Venezuela \\ https://orcid.org/0000-0002-8540-982X
}

\section{Resumen}

El propósito de este ensayo es reflexionar acerca del aprendizaje de la tecnología en la etapa escolar venezolana. Metodológicamente se procesó la información a partir de los planteamientos teóricos de Coll y Monereo (2008); Tizón (2008); entre otros, que están relacionados con la praxis pedagógica utilizada en la implementada en la apropiación de los contenidos programáticos con la incorporación de las Tecnologías de Información y Comunicación (TIC). Se determinó a modo conclusivo, que el proceso de generar cambios innovadores en los contenidos curriculares debe trascender las aulas de clase, en la búsqueda de promover en los docentes la responsabilidad de participar en las transformaciones pedagógicas escolares pertinentes para diseñar una arquitectura de las TIC, que apoye la enseñanzaaprendizaje requerida por los entornos socioeducativos globales.

Palabras clave: aprendizaje; tecnología; escolar.

Cómo citar este ensayo:

López, L. (2020). Aprendizaje de la Tecnología en la Etapa Escolar Venezolana. Revista Scientific, 5(15), 350-361, e-ISSN: 2542-2987. Recuperado de: https://doi.org/10.29394/Scientific.issn.2542$\underline{2987.2020 .5 .15 .18 .350-361}$

Fecha de Recepción: 30-05-2019
Fecha de Aceptación:

01-10-2019
Fecha de Publicación:

05-02-2020 


\title{
Technology Learning in the Venezuelan School Stage
}

\begin{abstract}
The purpose of this essay is to reflect on the learning of technology in the Venezuelan school stage. Methodologically, the information was processed based on the theoretical approaches of Coll and Monereo (2008); Tizón (2008); among others, which are related to the pedagogical praxis used in the one implemented in the appropriation of programmatic contents with the incorporation of Information and Communication Technologies (ICT). It was conclusively determined that the process of generating innovative changes in curricular content must transcend classrooms, in the search to promote in teachers the responsibility of participating in the pertinent educational pedagogical transformations to design an ICT architecture, that supports the teaching-learning required by the global socio-educational environments.
\end{abstract}

Keywords: learning; technology; school.

How to cite this essay:

López, L. (2020). Technology Learning in the Venezuelan School Stage. Revista Scientific, 5(15), 350-361, e-ISSN: 2542-2987. Recovered from: https://doi.org/10.29394/Scientific.issn.2542$\underline{2987.2020 .5 .15 .18 .350-361}$

Date Received:

30-05-2019
Date Acceptance:

01-10-2019
Date Publication:

05-02-2020 


\section{Introducción}

Las transformaciones que está atravesando el mundo contemporáneo en materia social, económica, política y cultural están vinculadas con una formación integral que debe desplegarse desde sus primeras etapas de escolaridad en el contexto de una sociedad compleja cada día más globalizada.

En este sentido, los docentes de todas las modalidades y niveles del sistema educativo deben poseer competencias tecnológicas orientadas a desplegar una praxis pertinente con las demandas de un proceso educativo globalizado por los avances de las ciencias y las Tecnologías de la Información y la Comunicación (TIC).

El proceso educativo tecnológico, hace ineludible, el compromiso de desplegar una praxis pedagógica dirigida a promover aprendizajes significativos en la Etapa escolar, porque en este nivel, se realiza debe realizar la alfabetización en todas las áreas del conocimiento y en especial en los ámbitos digitales con los cuales interactúan los educandos dentro y fuera de las aulas de clases.

La construcción de conocimientos significativos debe estar dirigida a generar en los escolares un progreso integral en los aprendizajes significativos. Por ello, la alfabetización cognoscitiva tecnológica en la etapa escolar debe estar asociada a los procesos renovadores del quehacer pedagógico en la sociedad del conocimiento.

Los planteamientos introductorios en este ensayo tienen el propósito abordar el aprendizaje de la tecnología en la etapa escolar venezolana, sustentada de un reconocimiento de los constructos presentados en documentos referenciales que estudian el proceso de la adquisición de dichas competencias, relacionados con la temática de las TIC, los cuales se despliegan a continuación. 


\section{Análisis de los Planteamientos Teóricos}

Las reformas educativas planteadas en Venezuela en los últimos veinte (20) años, redimensionan el proceso educativo desde sus concepciones filosóficas expuestas en los principios que sustentan las prácticas formativas destinadas a promover el aprendizaje significativo, con el fin de descubrir constructos teóricos destinados a ampliar las fronteras de la innovación educativa tecnológica en la Etapa escolar.

De este modo, las exigencias de innovación tecnológica en los ámbitos escolares, tiene un sustento en los fundamentos legales establecidos en la Constitución de la República Bolivariana de Venezuela (1999), en el artículo 103, donde se establece que: "toda persona tiene derecho a una educación integral de calidad, permanente en igualdad de condiciones y oportunidades" (pág. 17). Es precisamente esta premisa una de las que orientan las políticas educativas destinadas al desarrollo humano, social, profesional, premisas consustanciadas con la calidad formativa de los ciudadanos.

Asimismo, las premisas del Ministerio del Poder Popular para la Educación (2007a), establecidas en el Diseño Curricular, destaca un eje integrador en las políticas formativas en los niveles y modalidades educativas donde establece que: "impulsará el dominio de las nuevas tecnologías con un enfoque social y como herramienta de trabajo para el manejo y apropiación de la información" (pág. 13). Esta premisa está orientada al desarrollo de potencialidades de los educandos, en su intención de formar usuarios de la ciencia y tecnología en función del bienestar de su comunidad.

El aprendizaje de las tecnologías requiere de una alfabetización electrónica, de acuerdo con Tejedor (2009): implica desarrollar entre otros aspectos, las oportunidades de adquirir información además de "crear valores, resolver problemas y colaborar con otros medios del uso de las tecnologías electrónicas y de las redes" (pág. 27). De ahí, que la gestión escolar, en estos momentos de globalización, debe generar una innovación tecnológica que 
cognoscitivamente sea significativo para los educandos, además, responda a las exigencias de la sociedad global contemporánea.

Igualmente, la reforma de la Ley Orgánica de Educación (LOE, 2009), establece en el Art. 6, en su numeral 1, literal g, que "las condiciones para la articulación entre la educación y los medios de comunicación, con la finalidad de desarrollar el pensamiento crítico y reflexivo" (pág. 3). Por ello, las gestiones del proceso pedagógico entre los que se destacan la coordinación de las políticas educativas destinadas al uso y desarrollo de TIC.

Según Woolfolk (2010), señala que "las computadoras, los teléfonos celulares, las agendas electrónicas, los iPod, los videos juegos y otros medios digitales han cambiado la vida de todos" (pág. 336). Por ello, las tecnologías activan aspectos cognitivos emergentes de las construcciones generadas entre las relaciones entre los saberes previos, las necesidades, motivaciones y experiencias adquiridas por los educandos. Asimismo, Guerrero (2014), sostiene que: "el aprendizaje significativo, se refiere a que el proceso de construcción de significados es el elemento central del proceso de enseñanzaaprendizaje" (pág. 6). Por ello, las Tecnologías de Información y Comunicación (TIC), juegan un rol determinante en las experiencias pedagógicas en todos los niveles del sistema educativo.

En este cometido educativo, en las aulas de clase de algunas escuelas venezolanas se han introducido la enseñanza de las tecnologías, al ser utilizadas las computadoras "Canaima", como herramientas de enseñanza constructivistas para la creación de experiencias de aprendizaje significativo que responda a los intereses y necesidades de los educandos. De este modo, en algunas instituciones de primaria se introdujo la alfabetización electrónica, generando cambios pedagógicos en sus actividades dentro y fuera del aula de clases, por ello, Tizón (2008), explica que: "las nuevas tecnologías como recurso demandan de los centros educativos una disposición diferente del espacio y del tiempo, que no incida en el trabajo individual de transformación" 
(pág. 23). En este sentido, las herramientas tecnológicas, facilitan las posibilidades del aprendizaje por descubrimiento, por ello, el espacio virtual no tiene limitaciones de tiempo y espacio, contribuyendo a la transversalidad del conocimiento, y generando la asimilación de nuevos contenidos significativos.

Estas premisas formativas, sugieren de manera implícita que la praxis educativa para responder a las demandas de las Tecnologías de Información y Comunicación (TIC), debe percibir la realidad formativa en los ámbitos de aprendizaje significativo de los educandos, razón por la cual, se hace prescindible la adquisición de estas herramientas tecnológicas.

Sin embargo, sostiene Coll y Monereo (2008), que las Tecnologías de Información y Comunicación (TIC): "no sólo proporcionan una serie de herramientas tecnológicas, de recursos, de aplicaciones de software informático y telemático que sus usuarios potenciales pueden utilizar para aprender y enseñar" (pág. 86); sino que además, representa un conjunto heterogéneo de técnicas, sistemas, aparatos electrónicos, máquinas inteligentes, además de las redes tecnológicas, lo cual implica, un reconocimiento de la complejidad en la apropiación y dominio en la praxis educativa.

De igual manera, la alfabetización tecnológica tiene varios exponentes, entre los que se Gutiérrez (2003); Snyder (2004); Monereo (2005): manifiestan la importancia de la adquisición de destrezas en el uso inteligente de las TIC. Asimismo, hay que reconocer la importancia de la adquisición de los conocimientos pertinentes para responder a las demandas educativas a través de la integración curricular escolar, de manera más coherente con las exigencias de la sociedad global.

Desde esta orientación formativa, la alfabetización tecnológica debe enfatizar la importancia de un cambio en los contenidos escolares que tome en cuentas los requerimientos del perfil formativo de los educandos para adquirir los conocimientos con el aprovechamiento de las TIC en el quehacer 
pedagógico. Por ello, una praxis innovadora debe ser capaz de favorecer la construcción de aprendizajes significativos orientada al desarrollo de competencias para el tratamiento de la información y la adaptación de los elementos digitales en pro de alcanzar la calidad educativa.

En correspondencia con los planteamientos que anteceden, la innovación educativa, está estrechamente relacionada con lo expuesto por es importante implementar herramientas que sean prácticas, además de técnicas que permitan generar cambios, en los procesos y servicios educativos. Por este motivo, la Etapa escolar, es un ámbito ideal para propiciar la alfabetización tecnológica, sustentadas en las premisas educativas vigentes en Venezuela.

De este modo, las directrices de las políticas educativas en relación a las TIC, según Cabello (2008), citado por Pérez y Quintero (2016): "se torna más compleja pues no solamente se consideran aspectos vinculados con contenidos sino se agregan las múltiples posibilidades generadas en la interactividad" (pág. 201). Sin embargo, no se traducen automáticamente en cambios en las prácticas pedagógicas en las aulas de clase.

De este modo, las propuestas formativas de la etapa escolar venezolana, se requiere de un sustento curricular, que tome en cuenta las habilidades instrumentales de la gestión pedagógica de los docentes, las cuales están explicitadas en las acciones reproductivas de los contenidos programáticos de la etapa escolar que promueva en la praxis educativa un aprendizaje significativo de los mismos.

Por ello, la praxis educativa en las aulas de clase de la etapa escolar debe impactar en la construcción social de unos aprendizajes significativos apoyados en las TIC, para responder a los intereses, concepciones y actitudes previas del educando, a fin de fomentar las experiencias innovadoras que conlleven a la construcción de conocimientos relevantes para responder a las demandas de la sociedad. Así quedó expuesto por el Ministerio del Poder 
Popular para la Educación (2007b), establece que:

La incorporación de las TIC's en los espacios y procesos educativos, contribuye al desarrollo de potencialidades para su uso; razón por la cual el SEB, en su intención de formar al ser social, solidario y productivo, usuario y usuaria de la ciencia y la tecnología en función del bienestar de su comunidad (pág. 58).

De esta manera, los procesos generados por la innovación, lo cual se convierten en proyectos de perfeccionamiento del quehacer escolar, dentro de acciones pedagógicas que progresivamente articulan teoría y la práctica, al mismo tiempo que constituyen según Rivas (2019): "una respuesta a la innovación pedagógica, este factor determina el desarrollo personal del estudiante de sus experiencias formativas en la escuela" (pág. 38). Así las posibilidades de generar cambios pedagógicos, actúa de manera paralela con la motivación del estudiante por aprender de manera significativa e incide en la efectividad intelectual ante la posibilidad de promover una enseñanza pertinente, pues ambas son importantes en el proceso escolar.

En tal sentido, los procesos de innovación tecnológica en el quehacer pedagógico, desde la integración de estrategias didácticas a través del uso de las TIC, introduce conocimientos, competencias y actitudes en los docentes que conllevan a un quehacer pedagógico más acorde a las exigencias de la sociedad del conocimiento, condición indispensable de los procesos formativos destinados a estimular la enseñanza-aprendizaje escolar.

En el ámbito de aprendizaje significativo escolar, la innovación tecnológica integrada a los procesos didácticos del docente representa una estrategia constructivista consustanciada con los nuevos enfoques pedagógicos para abordar los requerimientos de la sociedad del conocimiento; de este modo la formación en el nivel de educación básica representa para este propósito, un punto de partida congruente con la globalización de los saberes contemporáneos. 


\section{Conclusiones}

Una vez culminada la exploración de los constructos teóricos, se puede concluir que el aprendizaje de las tecnológicas en la etapa escolar venezolana requiere estudiar los nuevos paradigmas didácticos para asumir una visión tecno educativa que responda a los requerimientos de una sociedad global.

El potencial educativo, de las tecnologías de información y comunicación, representa un reto pedagógico en la sociedad de la información y el conocimiento tecnológico adquirido en la etapa escolar venezolana. Por ello, se debe desarrollar una praxis educativa que responda a las expectativas, intereses y necesidades de los educandos para mantener una dinámica de interacción, que despierte la motivación que conlleve al aprendizaje tecnológico significativo.

En este sentido, la alfabetización tecnológica en la etapa escolar venezolana contribuye una oportunidad de innovación en la praxis pedagógica mediada por las TIC, lo cual representa desplegar los conocimientos pertinentes con las demandas de los avances científicos y tecnológicos en el marco del Siglo XXI.

Desde esta perspectiva, el aprendizaje significativo de la tecnología representa una alternativa de optimización de la calidad en el proceso formativo escolar que en Venezuela está enmarcada en las políticas educativas, además de responder a las exigencias de la sociedad del conocimiento.

Finalmente, los contenidos curriculares deben ser adaptados para innovar el quehacer pedagógico del docente, para asumir su responsabilidad de innovar con estrategias educativas que conlleven a una nueva arquitectura en los procesos de enseñanza-aprendizaje en pro de generar aprendizajes significativos acordes con las exigencias de los entornos globales en los cuales hacen vida los educandos. 


\section{Referencias}

Coll, C., \& Monereo, C. (2008). Psicología de la Educación Virtual. Aprender y Enseñar con las Tecnologías de Información y Comunicación. ISBN: 978-84-7112-519-4. Madrid, España: Ediciones Morata, S.L.

Constitución de la República Bolivariana de Venezuela (1999). Gaceta Oficial Extraordinaria N. ${ }^{\circ} 36.860,30$ de diciembre. Caracas, Venezuela: Asamblea Nacional Constituyente.

Guerrero, M. (2014). Metodologías Activas y Aprendizajes por Descubrimiento. Las TIC y la Educación. ISBN: 978-84-15878-15-5. Albacete, España: Marpadal Interactive Media, S.L.

Gutiérrez, A. (2003). Alfabetización Digital. Algo más que Ratones y Teclas. ISBN: 84-7432-877-2. Barcelona, España: Editorial Gedisa.

LOE (2009). Ley Orgánica de Educación. Gaceta Oficial № 5.929 Extraordinario del 15 agosto. República Bolivariana de Venezuela: La Asamblea Nacional. Recuperado de:

https://www.urbe.edu/portal-biblioteca/descargas/Ley-Organica\%20deEducacion.pdf

Ministerio del Poder Popular para la Educación (2007a,b). Currículo Nacional Bolivariano. Diseño Curricular del Sistema Educativo Bolivariano. ISBN: 978-980-218-281-7. Caracas, Venezuela: Fundación Centro Nacional para el Mejoramiento de la Enseñanza de Ciencia CENAMEC. Recuperado de:

\section{https://www.oei.es/historico/quipu/venezuela/dl 908 69.pdf}

Monereo, C. (2005). Internet y Competencias Básicas. Aprender a

Colaborar, a Participar, a Comunicarse, a Aprender. ISBN: 84-7827373-5. Barcelona, España: Editorial Graó.

Pérez, W., \& Quintero, E. (2016). Competencias Docentes para la Construcción del Conocimiento de los Estudiantes de Educación 
Primaria. Revista Scientific, 1(1), 190-211, e-ISSN: 2542-2987. Recuperado de:

https://doi.org/10.29394/scientific.issn.2542-2987.2016.1.1.11.190-211 Rivas, A. (2019). Factores que producen diferencias significativas en el aprendizaje de los estudiantes que egresan de sexto grado. Revista Scientific, 4(12), 28-46, e-ISSN: 2542-2987. Recuperado de: https://doi.org/10.29394/Scientific.issn.2542-2987.2019.4.12.1.28-46

Snyder, I. (2004). Alfabetismos Digitales. Comunicación, Innovación y Educación en la Era Electrónica. ISBN: 84-9700-169-9. Málaga, España: Ediciones Aljibe.

Tejedor, S. (2009). La Enseñanza del Ciberperiodismo. De la Alfabetización Digital a la Alfabetización Ciberperiodística. ISBN: 978-84-96082-46-5. Sevilla, España: Comunicación Social, Ediciones y Publicaciones.

Tizón, G. (2008). Las TIC en Educación. ISBN: 9781409227236 . Estados Unidos: Lulu Press, Inc.

Woolfolk, A (2010). Psicología Educativa. ISBN: 978-607-442-503-1. México: Prentice Hall (Pearson Educación). 


\section{Luisana Sleny López Alvarado \\ e-mail: sleny 82@hotmail.com}

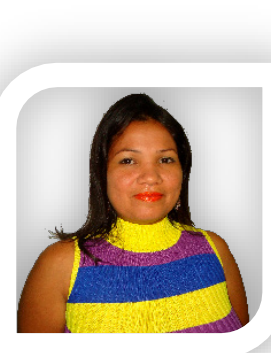

Nacida en San Felipe Estado Yaracuy, Venezuela, el 18 de julio del año 1982. Licenciada en Ciencias del Deporte en la Universidad Experimental de Yaracuy (UNEY); Maestría en Ciencias, Mención Orientación de la Conducta en el Centro de Investigaciones Psicológicas Psiquiátricas Sexológicas (CIPPSV) de Venezuela. Actualmente, Docente de aula del Ministerio del Poder Popular para la Educación 2014-2017. Investigadora en Ciencias de la Educación.

El contenido de este manuscrito se difunde bajo una Licencia de Creative Commons ReconocimientoNoComercial-Compartirlgual 4.0 Internacional 